\title{
ESTABLISHING NEW FOUNDATIONS FOR THE USE OF REMOTELY-PILOTED AIRCRAFT SYSTEMS FOR CIVILIAN APPLICATIONS
}

\author{
M. Balsi ${ }^{1, *}$, S. Prem ${ }^{2}$, K. Williame ${ }^{3}$, D. Teboul ${ }^{4}$, L.Délétraz ${ }^{5}$, P.I. Hebrard Capdeville ${ }^{6}$ \\ ${ }^{1}$ DIET, La Sapienza University, Rome, Italy - marco.balsi@uniroma1.it \\ ${ }^{2}$ Viasat, Lausanne, Switzerland - sam.prem@viasat.com \\ ${ }^{3}$ Unifly, Antwerp, Belgium - koen.williame@unifly.aero \\ ${ }^{4}$ Connectiv-IT, Paris, France - dteboul@connectiv-it.com \\ ${ }^{5}$ Skyguide, Géneve, Switzerland - laurent-deletraz@skyguide.ch \\ ${ }^{6}$ M3 Systems, Toulouse, France - inti.hebrard@m3systems.eu
}

KEY WORDS: UAV, BVLOS, satellite communications, U-space, UTM, infrastructure survey, corridor mapping

\begin{abstract}
:
Skyopener is a project funded by the EU through the European GNSS Agency (GSA) in the framework of the Horizon 2020 program. Skyopener's goal is contributing to the roadmap for the integration of civil Remotely Piloted Aircraft Systems (RPAS) into nonsegregated airspace, by providing and testing enabling technologies, in particular with reference to European initiative U-Space, aimed at establishing regulations and infrastructure for integration of unmanned aviation into shared airspace. The main outcomes of the project include: implementing and testing a reliable and secure redundant air-ground communication link, based on satellite and $3 \mathrm{G} / 4 \mathrm{G}$ networks; integrating the mission management system and ground station with a UTM (Unmanned aerial system Traffic Management) client, and experimenting UTM services being deployed by one of the partners; demonstrating technical and economic feasibility of long- range missions beyond visual line of sight (BVLOS) by executing corridor mapping on a high-voltage powerline, and airport area surveys (e-TOD: electronic-Terrain Obstacle Database).
\end{abstract}

\section{INTRODUCTION}

\subsection{Overview and motivation}

Remotely Piloted Aircraft Systems (RPAS) have a potential for a wide range of civil applications that would benefit European citizens and industry. As a result, the demand to operate RPAS over long ranges is drastically increasing. However, the regulation regarding the use of RPAS in civil airspace is not yet ready to enable operations beyond visual line of sight (BVLOS). In Europe, the Single European Sky initiative (SESAR Joint Undertaking) is promoting the development of U-Space (https://www.sesarju.eu/U-space), a regulatory and technical framework for gradually extending operability of drones into shared airspace, also based on the Unmanned aerial systems Traffic Management (UTM) system.

The Skyopener project is funded by the EU through the European GNSS Agency (GSA) in the framework of the Horizon 2020 program. Skyopener's goal is testing the use of RPAS for civilian applications to contribute to the roadmap for the integration of civil RPAS into non-segregated airspace.

In this context, the use of EGNOS (European Geostationary Navigation Overlay System) and Galileo is a major opportunity to provide enhanced navigation services in terms of integrity and positioning accuracy. EGNOS is the first pan-European satellite navigation system. It augments the US GPS satellite navigation system and makes it suitable for safety-critical applications such as flying aircraft or navigating ships through narrow channels. Sometimes called the 'European GPS', Galileo provides accurate positioning and timing information using data from its 18 satellites. When the constellation becomes fully operational in
2020, it will provide high precision and reliability, and when used together with GPS it will allow receivers to determine position at few centimeter accuracy (ESA, 2019). For these reasons, Rollout of Galileo services is being watched with great interest also in the RPAS world (GSA, 2019)

Skyopener is developing and testing a system and operational processes aimed at reducing all categories of risks associated with RPAS. The system will allow Air Navigation Service Providers (ANSPs) to monitor RPAS operations taking place in the very low level (VLL) airspace, which is below $500 \mathrm{ft}$ above ground level. This is done by implementing a UTM (Unmanned aerial system Traffic Management) system that interacts with Air Traffic Management (ATM).

Currently, no country allows unrestricted flight of RPAS in shared airspace. BVLOS flights are being authorized in many countries, under limitations aimed at enhanced safety, and normally within restricted airspace under NOTAM (Notice To AirMen). The Joint Authorities for Rulemaking in Unmanned Systems (JARUS) organization is a group of experts gathering regulatory expertise from all around the world. At present, 59 countries, as well as the European Aviation Safety Agency (EASA) and EUROCONTROL, are contributing to the development of JARUS work products. In particular, JARUS has developed, and is constantly updating, a standardized framework for security assessment with the purpose of flight authorization request, the guidelines for Specific Operations Risk Assessment (SORA - JARUS, 2019), that Aviation Authorities worldwide are using in authorization procedures.

The project will also demonstrate two applications, where drones are not applied yet, due to regulations constraints. The first is

* Corresponding author 
surveillance of linear infrastructure, such as corridor mapping, for powerlines, railways, pipelines. The second is mapping airport area obstacles in the framework of the e-TOD (electronicTerrain Obstacle Database) standard.

\subsection{Goals}

The number and density of RPAS operations in non-segregated airspace is anticipated to increase. Skyopener holds the promise of being an enabling asset for ANSPs (Air Navigation Service Providers) and UTM service suppliers by developing elements required to operate RPAS in the "Specific" operational category, as described in European Aviation Safety Agency (EASA) Notice of Proposed Amendment (NPA) 1705-05 A \& B (EASA, 2017)

Dissemination of project results will contribute to regulation drafting, in particular the overall concept defined in the "RPAS ATM Concept of Operations" published by EUROCONTROL (2017). Specifically, it will contribute to very-low-level (VLL) airspace operations where RPAS must seamlessly integrate with manned aircraft operating under Visual Flight Rules (VFR) under specific conditions. These are prescribed by the National Aviation Authorities (NAA) and can vary from State to State, e.g. police helicopters, military aircraft, balloons, gliders, training aircraft, fire-fighting aircraft, and ultra-light aircraft. However, RPAS operating in this airspace do not have to conform to either Instrument Flight Rules (IFR) or Visual Flight Rules (VFR), as set in International Civil Aviation Organization (ICAO) Annex 2. Skyopener shall perform its demo flights in Switzerland, after test flights in France, and focuses specifically on the European regulation process of EASA. However, concepts and techniques developed for this project are directly applicable worldwide.

Moreover, the conclusions of the Skyopener project will contribute to the work of the SESAR Joint Undertaking (JU), as indicated in the recently published U-Space Blueprint that sets out the vision for the U-space. U-space aims at enabling complex drone operations with a high degree of automation to happen in all types of operational environments, particularly in an urban context. When fully deployed, a wide range of drone missions that are currently being restricted will be possible thanks to a sustainable and robust European ecosystem that is globally interoperable.

Skyopener culminates in a long-range survey demo that shall be performed in fully automatic flight mode beyond visual line of sight. The focus of the demo is on security and reliability of the communications link, and on an operational test of Unifly's UTM, interfaced with the system's ground station and active at the competent air traffic control site. Skyopener's demo does not include detect-and-avoid features, and the flight is preprogrammed before the flight, and possibly changed remotely during the flight. Therefore, flight mode is not fully autonomous, but the features being demonstrated are instrumental enabling technologies for BVLOS flight, both automatic and autonomous.

\section{Specific objectives}

The detailed objectives of Skyopener are the following:

Improvement of reliability and security of navigation systems used on RPAS. Skyopener will test Galileo signals as a complement to GPS to show improvement of reliability of the overall navigation. This will improve the positioning robustness and accuracy and mitigate against the risk of GPS signal loss and collision. The basic assessment possible with existing technology, employed in Skyopener's drone, is checking signal integrity, and estimating positioning error of the Galileo signal alone. However, the real substantial improvement is brought about by Galileo when used together with other constellations, in particular GPS, in a Precise Point Positioning (PPP) framework (Basile et al, 2018).

Integration of Satellite Communication to increase the reliability of the RPAS communication systems. Radio communication systems on board RPAS usually provide $80-90 \%$ availability. SatCom systems provide much better availability, typically $99 \%$. Skyopener is testing a system that will significantly enhance the reliability and the availability of the CNPC (Control of NonPayload Communication). It will combine radio and satellite link systems with hot redundancy, thus increasing availability up to $99.9 \%$.

Testing a UTM system dedicated to RPA VLL operations with interactive surveillance feature. Skyopener is testing the UTM system developed by one of its partners (Unifly) at U-Space level 2, and partly level 3, that allows management of flight authorization procedures and real-time monitoring of air traffic from the ground station, and will also demonstrate the capability for the ANSP to receive data from the RPAS to show the aircraft's position in real time. Information (aeronautical, manned aviation positions) from the ANSP is also shared to the pilot through UTM integration, completing the situational awareness of the drone pilot.

Improvement of information processing and presentation on $R P A S$ to reduce the risk of human error. Skyopener is testing the user interface at the level of the Remote Pilot in the RPAS, developing a more user-friendly interface for the pilot.

Testing the RPAS mission payload and software package for $e$ TOD services and linear surveillance operations beyond line of sight. Skyopener is testing an integrated mission payload that includes remote sensing (LiDAR), a high definition visiblespectrum camera, an IR camera, and direct geo-referencing with satellite navigation. The goal is to provide accurate information about obstacles within the e-TOD (Electronic Terrain and Obstacle data) services geographical boundaries, as well as objects (trees, temporary structures, etc.) that intrude into linear infrastructure corridors, and emergency or post-emergency surveys (landslides, flooding, collapse, short-circuits, etc.) of critical infrastructure.

\section{ARCHITECTURE}

According to the U-Space blueprint (SESAR JU, 2018), "the progressive deployment of U-space is linked to the increasing availability of blocks of services and enabling technologies", therefore it will be implemented gradually, according to interconnected technological and regulatory developments.

Skyopener contributes enabling technologies for levels 1, 2 and 3 , aiming at demonstrating technology readiness.

Level 1 (foundation) services include:

- e-registration,

- e-identification,

- geofencing;

while level 2 (initial) services support the management of drone operations, and may include:

- $\quad$ flight planning,

- flight approval,

- tracking,

- airspace dynamic information, 
- $\quad$ procedural interfaces with air traffic control.

At level 3 (advanced services), Skyopener contributes in particular to conflict detection.

Key principles of U-Space are all addressed by the project, in particular by the following provisions:

- Ensuring safety of airspace users and people on ground, applying a risk-based approach, consistently with the Specific Operations Risk Assessment (SORA) procedure developed by JARUS. At this stage of the project, the demo flights will comply to stricter security criteria than what will be allowed in the future at level 2 of U-space, e.g. by avoiding overflight of towns, or critical infrastructure such as railroads, except for rapid transversal crossings, or by maintaining radio line-of-sight as a backup measure, even if this will not be used unless an unexpected emergency happens.

- Minimizing costs of service provision, deployment, operation, by leveraging on existing infrastructure and services, including GNSS, and mobile as well as satellite communication networks, as well as employing off-theshelf technology where available, possibly upgrading and/or adapting it to comply with applicable safety and performance requirements.

- Enabling shared use of airspace by manned and unmanned vehicles guaranteeing visibility of all traffic on the same platform to all users and air traffic controllers.

Skyopener system architecture is described in Fig. 1.The main components of the system are:

- the RPA, capable of fully autonomous flight, connected to satellite (L-band) network for high reliability, and to $3 \mathrm{G} / 4 \mathrm{G}$ cellular network for high throughput, carrying payload for mission execution and using FLARM for separation from light air traffic, and advanced GNSS receiver for precision and reliability;

- the ground station (Remote Pilot Station, or RPS), connected to the RPA through satellite and cellular network, as well as to air traffic management through highthroughput Ka-band satellite network;

- the UTM system, in perspective a public service, guaranteeing monitoring and separation of unmanned air traffic, and interaction with existing manned Air Traffic Management (ATM).

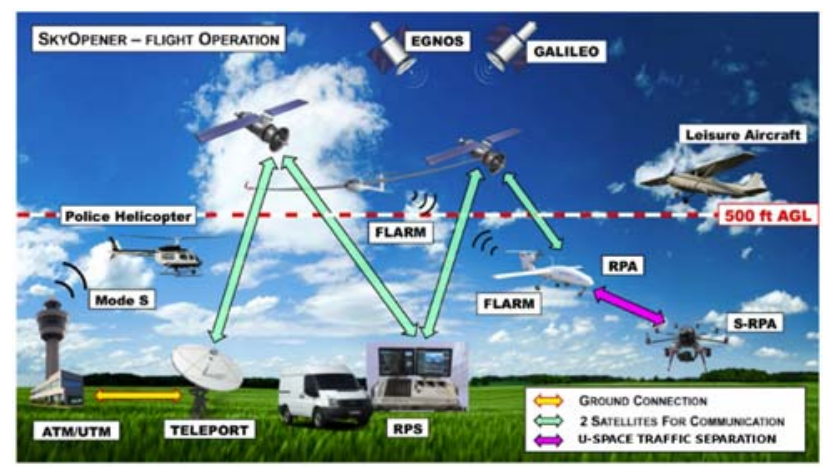

Figure 1. Skyopener system architecture.

\subsection{RPA}

The RPA employed in the project is a fixed-wing drone by Boréal, Toulouse, France (www.boreal-uas.com), Fig. 2, set up and operated by sister company M3 Systems, Toulouse, France (m3systems.net). It has a wingspan of $4.2 \mathrm{~m}$, take-off weight less than $25 \mathrm{~kg}$, and is capable of carrying up to $5 \mathrm{~kg}$ payload (about $43 \mathrm{dm}^{3}$ volume). It is propelled by a gasoline engine, with a maximum endurance of $10 \mathrm{~h}(1000 \mathrm{~km})$, with cruise speed between 65 and $130 \mathrm{~km} / \mathrm{h}$. It is launched by a catapult, and lands on a short range on its belly.

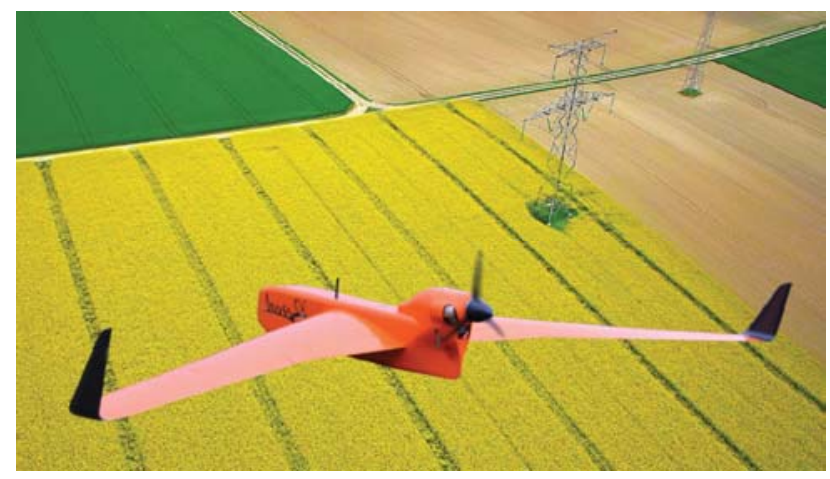

Figure 2. The Boréal drone

The drone is equipped with a proprietary autopilot, capable of fully automatic flight control. It relies on a multi-constellation GNSS receiver, enabled for EGNOS and Galileo. Besides being used directly by the autopilot for navigation, Galileo signals, along with those proceeding from other constellations, will also be recorded and used for survey data georeferencing. In this way, the project will provide valuable quantitative evaluation of the increased precision expected from Galileo.

The autopilot is connected to the ground station by a hotredundant command-and-control (C2) link, based on L-band satellite networking, provided by Viasat, Lausanne, Switzerland, that ensures very high reliability (typically 99\% of time, compared to no more than $90 \%$ for other types of connections), supported by $3 \mathrm{G} / 4 \mathrm{G}$ cellular connection, for high throughput (in particular for sensor data transmission) and backup. A VHF radio is used only in (relatively short) radio line of sight, for direct remote control, that is normally preferred for take-off and landing.

A mission computer on board, interacting with the autopilot, manages on-board services, in particular payload sensors, controlling their operation, recording data and co-registering them with GNSS and AHRS (attitude) information, as well as generating alert or alarm messages, and transmitting sensor data (in particular images) to ground when needed.

\subsection{Ground Station and UTM}

The Ground Control Station is an essential part of the system to manage the mission and ensure safety. The Skyopener solution, developed by Connectiv-IT, Paris, France (www.connectivit.com), provides a user-friendly interface for mission planning and management, and interfaces with the UTM client developed and deployed by Unifly, Antwerp, Belgium (www.unifly.aero) to access its UTM platform. Unifly also participates in CORUS lead by Eurocontrol which is the SESAR JU project defining the conops (concept of operations) of UTM. In this framework, Unifly has been actively improving its UTM system, so that it is one of the most completely developed and deployed proposals 
worldwide. Unifly's UTM is deployed today by ANSP's and CAA's in Denmark, Germany, Austria, Belgium, Japan. It provides support for all level-2 U-Space services and most level3 U-Space services including collaborative ATC interface and tactical conflict resolution, managing the whole chain of flight planning, flight approval, tracking, collision detection, dynamic airspace information, and procedural interfaces with manned air traffic control (ATM). Of course, full deployment of the platform in individual countries or selected areas depends on the regulatory process still in progress and on implementation of $U$ Space deployment (or of equivalent service outside Europe), but Skyopener will demonstrate interaction with airport air traffic controllers and the ATM environment, thanks to collaboration of the Swiss civil aviation authority, Skyguide, Genève, Switzerland (www.skyguide.ch), and by using the ATM/UTM gateway developed within the project, leveraging both greenfield protocols and reusing ATM-industry standard protocols.

\subsection{Services, payload and demo applications}

While the focus of Skyopener is on demonstrating a complete system and environment for BVLOS operations, based on secure and reliable communication, and on integration with UTM, the demo flights of the project will also demonstrate feasibility and economic advantages of long-range/long-endurance unmanned missions. To this purpose, two significant applications shall be implemented, namely corridor mapping (survey of a stretch of high-voltage powerline), cfr. Matikainen et al., 2016, and airport area survey for e-TOD database population (survey of obstacles - ICAO, 2018).

Based on requirements of such applications, a suitable set of sensors was selected, including:

- a Yellowscan Surveyor Ultra LiDAR, based on a Velodyne VLP-32C sensor, capable of obtaining, with the typical flight profile employed $(80 \mathrm{~km} / \mathrm{h}, 70 \mathrm{~m} \mathrm{AGL})$, about 30 points $/ \mathrm{m}^{2}$ on ground, with two echos and $0.1 \mathrm{~m}$ accuracy;

- a camera for visible spectrum Allied Vision Prosilica GT6600 with 35mm-type color sensor, 29Mpixel, and $28 \mathrm{~mm}$ Canon lens $\left(33^{\circ} \mathrm{FOV}\right)$, 4shots/s maximum shutter frequency;

- a FLIR A65 IR camera, with $13 \mathrm{~mm}\left(45^{\circ} \mathrm{FOV}\right)$ lens;

The LiDAR is used as the main source for 3D surface reconstruction. Its multi-echo feature also allows for terrain mapping under vegetation cover. It is used to obtain accurate DTM, and detailed representation of objects above ground (buildings, structure, powerline pylons and wires, vegetation).

The visible spectrum camera is used mainly for production of high-definition orthophotographs, but also for line following in the powerline application, as a check for actual trajectory, and upon request it is possible to obtain images on the ground station in real time, e.g. when a direct visual check is required by the operator.

The IR camera is mounted for the powerline survey application, in order to detect hotspots denoting possible failures.

Optics for the cameras were chosen considering flight profile requirements (minimum safe operating speed, minimum safe altitude) and LiDAR characteristics and requirements, and optimizing the trade-off between swath and definition, also considering that some data are more relevant at shorter distance from the sensor, in particular power lines are closer to the RPAS during flight than ground.

Exploitation of data obtained in the demo surveys is performed by DIET, La Sapienza University of Rome, Italy (www.diet.uniromal.it) with the support of Oben srl, Sassari, Italy (www.oben.it).

The objective of corridor mapping for powerlines, is basically identifying any object (normally trees), that intrudes into a given distance from wires, depending on local regulations and practice, and on powerline voltage. DIET has developed a proprietary algorithm for automatic pylons and wires recognition and modeling, and detection of obstacles within a given distance from the wire (Fig. 3), tested on data provided by Oben and by Groupe, Fribourg, Switzerland, i.e. the Swiss energy distribution utility. Commercial solutions were also assessed. The project demo shall be performed in Switzerland in collaboration with Group-e.

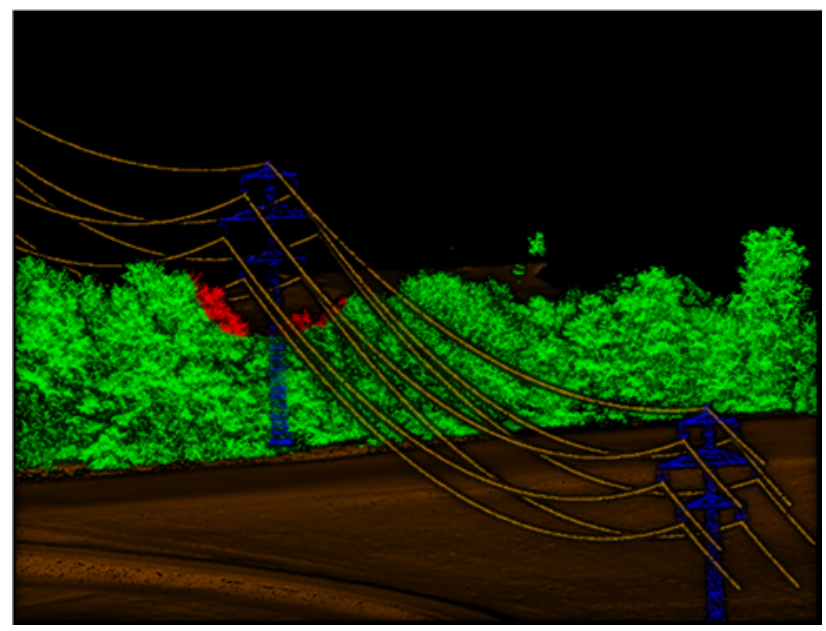

Figure 3. Detection of vegetation growing too close to a highvoltage powerline

For the e-TOD exercise, the object is detecting and accurately mapping any object rising higher than given thresholds in the airport area and its neighborhood. Such survey is mandatory, and updated periodically. Drone-based survey is especially useful in the inner areas inside and around the airport, where detail requirements are higher, and considerable savings may be obtained thanks to automation of the process, and operation within the airport during down times of air traffic. DIET developed the LiDAR data processing workflow for the project, based on GIS environment.

\section{EXPECTED RESULTS}

Integration Tests, including simulations of the demo exercise, to be performed in designated areas in the vicinity of Toulouse, France, were slightly delayed due to the authorization procedure. They shall be performed in the first half of April,

Demo flights shall be perfomed in the area of Payerne, Vaud, Switzerland in May, 2019, and results shall be ready for reporting at the UAV-g conference, and will be published in the project website (Skyopener.eu).

A UTM client shall be installed at the air traffic control site in Payerne airport, in collaboration with Skyguide and with military airport management. Another UTM client will be available at the system Ground Station, operated at takeoff/landing site at the airfield in Bellechasse, Fribourg, Switzerland.

The flight plan includes overfly of two stretches of the highvoltage powerline connecting Galmiz, Fribourg and Lucens, Vaud (fig. 4). It develops mostly in the CTR (Controlled Traffic 
Region) of the Payerne airport, but in order to simplify authorization it will avoid its ATZ (Aerodrome Traffic Zone). For the e-TOD exercise, the area of Bellechasse airfield shall be used.

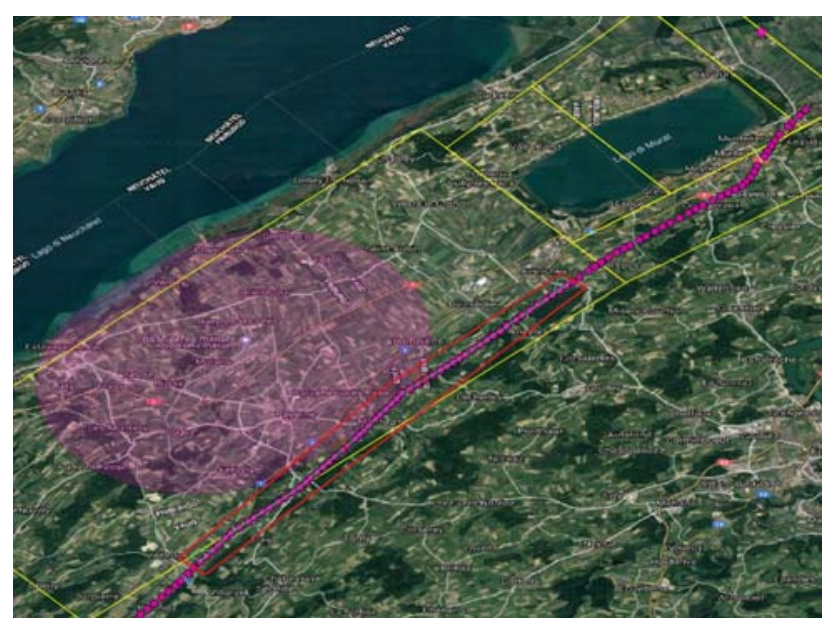

Figure 4. Demo flight area

\section{CONCLUSIONS}

Skypener demonstrates technology readiness for BVLOS missions in the framework of level-2 of U-Space. Main contributions to enabling technologies for such purpose are:

- implementation of a secure and dependable command-andcontrol communications links between RPAS and Ground Station, and from the latter to public infrastructure (UTM);

- integration of state-of-the-art UTM technology in the system architecture, demonstrating readiness for interaction with wide-area air traffic control, and providing smooth service request and provision chain.

These results were obtained under reasonable cost constraints, so that future long-range civil RPAS operations will compete strongly with manned-aircraft-based operations. Main reasons are the reduction in costs and simplified logistics, as well as enhanced quality and detail of data, thanks to the possibility of flying low, closer to survey targets. New applications for aerial survey and direct intervention (e.g. in maintenance, delivery, etc.), will be made possible in areas where large aircraft cannot fly.

The main challenge remaining is linked to safety guarantee and regulatory risk. Regulation authorities need to require very demanding proof of safety. This involves technological development such as demonstrated in Skyopener, but also extensive testing and certification. Another challenge is the regulating activity. Although expected to lead to an EU directive hopefully soon, this is necessarily a long-term process that does not allow for predicting immediate permission and management of large-scale commercial operation.

\section{ACKNOWLEDGEMENTS}

This work was supported by the European GNSS Agency (GSA) under grant 687352 - SKYOPENER - H2020-Galileo-GSA2014-2015/H2020-Galileo-2015-1

\section{REFERENCES}

Basile F., Moore T., Hill C., McGraw G., Johnson A., Multifrequency precise point positioning using GPS and Galileo data with smoothed ionospheric corrections, 2018. Proc. of 2018 IEEE/ION Position, Location and Navigation Symposium (PLANS), Monterey, CA, USA, Apr. 23-26, 2018. https://doi.org/10.1109/PLANS.2018.8373531

EASA (European Union Aviation Safety Agency), 2017. NPA 2017-05: Introduction of a regulatory framework for the operation of drones - Unmanned aircraft system operations in the open and specific category,

https://www.easa.europa.eu/document-library/notices-ofproposed-amendment/npa-2017-05 (28 March 2019)

ESA (European Space Agency), 2019. Galileo general introduction,

https://gssc.esa.int/navipedia/index.php/Galileo_General_Introd uction (28 March, 2019)

EUROCONTROL, RPAS ATM CONOPS. ver. 4.0, 2017. https://eurocontrol.int/sites/default/files/publication/files/Brochu re\%20RPAS\%20CONOPS.pdf (28 March, 2019)

GSA (European GNSS Agency), 2019. GSA highlights added value of EGNSS for drones at WATM 2019,

https:/www.gsa.europa.eu/newsroom/news/gsa-highlightsadded-value-egnss-drones-watm-2019 (28 March, 2019)

ICAO (International Civil Aviation Organization), 2018. Annex 15 - Aeronautical Information Services. http://www.icao.int (28 March, 2019)

Joint Authorities for Rulemaking in Unmanned Systems (JARUS) JAR doc 06 SORA (package), 2019. http://jarusrpas.org/content/jar-doc-06-sora-package - revised Mar. 2019 (28 March, 2019)

Matikainen L., Lehtomäki M., Ahokas E., Hyyppä J., Karjalainen J., Jaakkola A., Kukko A., Heinonen T., 2016. Remote Sensing Methods for Power Line Corridor Surveys. ISPRS Journal of Photogrammetry and Remote Sensing, Vol. 119, pp.10-31, https://doi.org/10.1016/j.isprsjprs.2016.04.011

SESAR Joint Undertaking, 2018. U-Space Blueprint. https://www.sesarju.eu/u-space-blueprint (8 Januar 2019) 